\title{
Ultrasound-Guided Core Biopsy of Breast Lesions in a Resource Limited Setting: Initial Experience of a Multidisciplinary Team
}

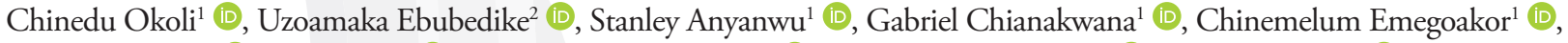

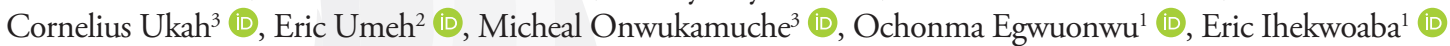

${ }^{1}$ Department of Surgery, Nnamdi Azikiwe University, Anambra, Nigeria

${ }^{2}$ Department of Radiology, Nnamdi Azikiwe University, Anambra, Nigeria

${ }^{3}$ Department of Pathology, Nnamdi Azikiwe University, Anambra, Nigeria

\section{ABSTRACT}

Objective: There is increasing tendency to multidisciplinary care of patients with of breast lesions. This study sought to evaluate the initial experience of the diagnostic arm of a new breast program in a resource limited setting.

Materials and Methods: In 2015, we commenced the pilot phase of an IRB-approved breast care protocol. As part of the protocol's diagnostic arm, an ultrasound-guided breast core biopsy training was implemented. Eligible patients were clinically evaluated and underwent CNB using 16G needle under US guidance. The procedure was rated by the participants and histopathological results compared with surgical specimens.

Results: Eighty six participants (18.22\%) with 113 palpable breast lesions completed the study. The diagnostic accuracy, sensitivity, and specificity were $94.44 \%, 92.86 \%$, and $95.83 \%$ respectively. Unweighted kappa- coefficient $(\mathrm{k})$ agreement between histopathology of core biopsy and surgically excised specimens, were $0.798(95 \%$ CI of $0.69-0.90)$ and 0.801 (95\% CI of $0.71-0.92)$ for benign and malignant breast lumps respectively. The procedure was well accepted and all the patients were willing to accept a repeat $\mathrm{CNB}$ and would recommend it.

Conclusion: Despite the prevailing challenges, co-ordinated team diagnosis is feasible and may result in the modest improvement in the diagnostic accuracy of breast lesions and patient satisfaction.

Keywords: Ultrasound-guided, core biopsy, palpable, breast

Cite this articles as: Okoli C, Ebubedike U, Anyanwu S, Chianakwana G, Emegoakor C, Ukah C, et al. Ultrasound-Guided Core Biopsy of Breast Lesions in A Resource Limited Setting: Initial Experience of A Multidisciplinary Team. Eur J Breast Health 2020; 16(3): 171-176.

\section{Introduction}

Globally, the multidisciplinary breast care team is responsible for breast cancer detection, diagnosis, as well as treatment and, are generally regarded as mandatory for the gold standard of care of breast diseases $(1,2)$. These multidisciplinary teams comprise specialists involved in all aspects of care of patients with breast diseases including medical, nursing, allied professionals, and diagnostic experts. This coordinated team approach to breast diseases diagnosis and management were formed after observational evidence showed better outcomes among patients treated by a team of different specialists for various common cancers (3).

This multidisciplinary model has usurped the surgeon-directed practice model prevalent in most resource-limited settings like Nigeria. Expert opinions suggest that collaborative efforts among these professionals during various phases of the diagnostic process and patient evaluation help to improve optimal patient care and eliminate system inefficiencies that may result in delayed breast cancer diagnoses. Hence, this approach is central to the delivery of a high-quality service.

Until recently, a tertiary healthcare provider in South-eastern Nigeria, offered full-time surgical oncology and breast care services using the surgeon-directed practice model. In this model, patients with breast lesions were directed to the surgeons who diagnose as well as perform either open or close biopsy. Prior implementation of freehand guided CNB (Core needle biopsy) was met by low diagnostic accuracy and several repeat open biopsy (4). This resulted in continual accrual of patients who neither had breast cancer nor required a breast operation in the operating list. Thus, worsening the waiting list of patients, decreased patient compliance, and further delayed the diagnosis of already late cases. These systemic delays are not only likely to impact negatively on the survival but also have a profound effect on the qual- 
ity of life of these patients. Breast cancer patients living in developing countries already have high case fatality rate (5) and severely impaired quality of life (6). With the increasing awareness of breast cancer, we believe that the number of biopsies will likely increase which will further compound the matter.

To mitigate these scheduling issues and to provide more rapid and a reliable alternative to the open surgical biopsy, the diagnostic arm of the breast program, commenced the use of ultrasound-guided core needle breast biopsy in a point-of-care setting run by a team of specialists. Though image guidance could be provided using mammographic, stereotactic and sonographic guidance, the latter modality was chosen because of the inherent advantages. These include low cost, absence of ionising radiation, full control of needle position in real-time, affordability, speed, availability, access to difficult places such as axilla or near the nipple as well as the possibility of sampling multiple lesions in one session (7). Furthermore, local anaesthesia and hematoma do not hide the lesion (non-calcified masses can obscure stereotactic equipment) (8).

To our knowledge, there is a paucity of studies reporting their experience of ultrasound (US)-guided biopsy of breast lesions in Nigeria by a multidisciplinary team. In this article, we aimed to analyse our initial results as well as discussing some of the initial challenges we encountered in the early phase of the procedure. The results of this study will help to underscore the benefits and feasibility of setting up multidisciplinary management of breast cancer patients in resourcelimited settings.

\section{Materials and Methods}

This study was a prospective study that involved 86 patients managed for breast diseases from January 2015 to October 2016 in the breast clinic of a university hospital. As part of the diagnostic arm of the protocol, the tumour board developed an ultrasound-guided percutaneous core-biopsy training programme for participating physicians. The surgeon performed biopsies with the sonographers assisting with image guidance and optimization.

\section{Subjects}

The patients were selected by purposive sampling from outpatient clinic attendees who fulfilled the study's inclusion criteria. The inclusion criteria included: i) presence of ultrasound-visible breast lesion; ii) subsequent surgery to remove the lesion or a minimum follow up of 1 year for patients with benign breast diagnosis on $\mathrm{CNB}$ who opted for no excision; and iii) a complete dataset. Exclusion criteria were: i) previous surgery of the target lesion; ii) Patients with non-palpable breast lesions. iii) Patients with a history of blood dyscrasia. iv) Patients with breast implants. v) Those who refused to give consent. vi) patients with benign breast lesions on CNB who were lost to follow up. This study was approved by the Ethics committee. It was carried out at no extra expense to the eligible patients who gave their informed consent. There was strict observation of the patients' confidentialities by using codes for reference, analysis and presentation of the results of this study. It also adhered to the tenets of the declaration of Helsinki for medical research in humans. Written consent was gotten from all the patients. Of the 181 patients with breast diseases that were assessed for eligibility, only 86 patients with 100 palpable breast lumps who completed the study were analysed. The clinical evaluation of both breasts was done by the attending consultant surgeon in the breast clinic while the
The dimensions of breast lumps were measured with a skin calliper and ultrasound Variables collected included age, clinical and Breast Imaging Reporting and Data System (BI-RADS ${ }^{\oplus}$ ) diagnosis. The primary clinical diagnosis was classified as malignant or benign. The variables were documented in a pre-structured proforma.

\section{Details of procedure}

Before each procedure, in the absence of pre-existing biopsy pack, the materials for biopsy were assembled consistently in a biopsy tray to ensure easy identification of all the materials needed for the procedure. This helped to minimise the risk of accidental needle stick injury and/ or contamination. Each biopsy tray comprised the following disposables: surgical glove to cover the ultrasound probe, tissue sample container, latex gloves, the semi-automated size 16- French gauge needle with $22 \mathrm{~mm}$ excursion (Egemen AC16150), lidocaine, 25 gauge needles, adhesive bandage, and size 11 scalpel blade; while the multi-use and bulk supplies included the following: formalin, iodine, ultrasound gel, sterile gauze, sterile drapes, and an autoclave drum.

Before each procedure, the biopsy needle was tested to ensure proper functioning before use. The patient was made to lie supine on a couch after exposure of the upper half of the body, with the side of the breast being evaluated elevated with a pillow whilst the ipsilateral shoulder was abducted with the hand placed palm up next to the head which will be turned away from the examiner. The breast skin was prepared initially using povidone-iodine and then isolated with a sterile drape and lubrication with a gel to facilitate ultrasound transmission. The radiologist then gently applied the transducer of Aloka prosound SSD350SX TM (Japan) ultrasound machine with a linear transducer (frequency of $7.5 \mathrm{MHz}$ ) and colour Doppler capability after covering with a sterile surgical glove, on the breast for the initial evaluation of breast lesion. The location of the lump was noted by the radiologist and this is confirmed by the surgeon. The Breast Imaging Reporting and Data System (BI-RADS ${ }^{\circ}$ ) category of the mass was then assessed using both longitudinal and transverse scans. To minimise inter-observer variability, the ultrasound classification of the breast lesion was done by two Consultant Radiologists using the BI-RADS ${ }^{\oplus}$ guidelines. Lidocaine 2\% (Jawa Lidocaine, Jawa group, Lagos, Nigeria) was injected superficially with a 25 -gauge needle, creating a subcutaneous wheal where the skin was to be entered. A small vertical skin incision was made with No 11 scalpel to aid in re-approximation of the defect during healing. The needle was then introduced into the lesion through the skin incision. The needle's position was confirmed by direct visualisation of the needle tip in the lesion on the ultrasound screen. The automated biopsy gun was then fired and the needle tip before and after biopsy firing was determined by longitudinal and orthogonal images to ensure that the needle was within the lesion.

Three to five cores of tissue were usually taken through the shortest route from the skin to the lesion. The core tissue sampling was done by the surgeon. Patients were monitored for complications including residual pain, breast hematoma, and pneumothorax. The samples were immersed in $10 \%$ formalin and transferred to the pathology laboratory where they were processed and paraffin-embedded. The appearance and behaviour of the formalin-fixed core samples were examined during the procedure to confirm that the targeted lesion was adequately sampled. The punctures were compressed for 5-10 min to control bleeding.

After the procedure, each patient was asked to assess the procedure using a 5 point Likert scale to rate the procedure. Patients were moni- 
tored for complications. The samples were then transferred to the pathology laboratory where they were processed and paraffin-embedded. The patients were followed up until the histopathological results of the CNB samples became available. The histopathological examinations were performed by 2 dedicated breast pathologists, and the results were categorized as malignant, high-risk, benign and indeterminate cases. Malignant results included invasive carcinoma and ductal carcinoma in-situ (DCIS). High-risk results included atypical ductal hyperplasia $(\mathrm{ADH})$, atypical lobular hyperplasia (ALH), lobular carcinoma in situ (LCIS), papillary lesions (intraductal papilloma and papilloma with atypia), phyllodes tumours and indeterminate cases. All other lesions were classified as benign findings. The radio-pathological concordance was performed between CNB results and imaging findings for each case. If the CNB result yielded malignant lesion, the patient underwent the respective surgery including mastectomy or wide local excision. In high-risk cases on $\mathrm{CNB}$, indeterminate cases, or radiopathological discordance, open surgical excision was performed. If the CNB yielded benign results concordant with sonographic imaging, patients were given the choice of excision biopsy or follow-up with imaging. All the patients who had concordant benign lesions and did not have surgery were followed up for a minimum of 3 years. The histopathologists analysing the open surgical specimens were blinded to the initial CNB histopathological results.

\section{Statistical analysis}

Statistical analysis used was the Statistical Package for Social Sciences version 21 (IBM SPSS Corp; Armonk, NY, USA). Results of categorical variables were expressed using Tables and Charts where appropriate. The mean age of the patients and sizes of the lumps were measured clinically and using ultrasound, were reported as the mean \pm standard deviation. The significance of the mean difference was determined using independent t-tests. The agreement between histopathological diagnosis of CNB and the histopathological result of surgically excised lumps was determined using unweighted kappa agreement tests with $95 \%$ confidence interval. A kappa score of 0 showed no agreement while a score of 1 showed perfect agreement. Statistical significance was inferred at a $\mathrm{p}<0.05$. The sensitivity, specificity, false-positive rate, false-negative rate, positive predictive value, negative predictive value and overall diagnostic accuracy were determined for ultrasound-guided CNB. The false-negative rate (FNR) and false-positive rate were calculated using the formulae (False negative) / (True Positive + False negative) and (False Positive) / (False Positive +True Negative) respectively.

\section{Results}

All the patients that had the ultrasound-guided CNB in this study were females. The age of the patients studied ranged from 12-78 years $($ mean $=40.12 \pm S D 13.81)$ with a median age of 39.0 years. The BIRADS categories of the breast lumps in this study ranged from 2-5 with categories 2 and 4 as the most common category while category 3 was the least frequent assessment (Table 1). The sizes of the breast lumps were estimated clinically and sonographically. The mean of the widest diameter (SD) measured clinically and with ultrasound were $65.2 \mathrm{~mm} \pm 0.6$ and $46.57 \mathrm{~mm} \pm 6.46$ respectively. Using a paired Ttest, the difference in mean was found to be statistically significant $(\mathrm{p}<0.001)$. Pathological examination of the CNB revealed that malignant lesions accounted for $40 \%(n=40)$ of $\mathrm{CNB}$ diagnosis, high-risk lesions accounted for $6.0 \%(n=6)$, and benign lesions accounted for 45 $(\mathrm{n}=45)$. Indeterminate cases were seen in $9.0 \%$ of $\mathrm{CNB}$ diagnosis as shown in Table 1 and characterized in Table 2.
The final surgical histological results were determined and shown in Table 1 . When the indeterminate cases were excluded, the sensitivity of CNB increased from $84.78 \%$ to $92.86 \%$, while the specificity decreased from $96.23 \%$ to $95.83 \%$. Other parameters are shown in Table 3. The agreement between the histopathological sub-classification of breast masses into definite histological entities using ultrasound-guided CNB specimens and open surgical specimens was analysed using unweighted kappa- coefficient and at a 95\% confidence interval. The $\mathrm{k}$ - coefficient values were 0.798 (95\% confidence interval of 0.69 to 0.90 ) and 0.801 ( $95 \%$ confidence interval of 0.71 to 0.92 ) with $\mathrm{p}<0.05$ for benign and malignant breast lumps respectively. This $\mathrm{k}$ value of $>0.7$ shows substantial agreement between the two pathological results and this was significantly greater than zero in this study $(0=$ no agreement). Comparisons of the results in this study with other series are shown in Table 4 (9-15).

The degree of acceptance of the procedure ranged from fair to excellent. Most of the patients without complications had rated the procedure as excellent while 2 patients with complications rated the procedure as fair. No patient rated the procedure poorly, however, all the patients said they would recommend the procedure to friends and relatives and would undergo the procedure in the presence of other breast diseases. Details are shown in Table 5.

\section{Table 1. Ultrasound grading/CNB histopathological diagnosis/ Open histological diagnosis}

$\begin{array}{lc}\text { Category } & \text { Frequency (\%) } \\ \text { BI-RADS® } & \\ 2 & 41(41) \\ 3 & 5(5) \\ 4 & 36(36) \\ 5 & 18(18) \\ \text { CNB histopathological diagnosis } & \\ \text { Indeterminate lesions } & 9(9) \\ \text { Benign } & 45(45) \\ \text { High risk } & 6(6) \\ \text { Malignant } & 40(40) \\ \text { Open histopathological diagnosis* } & \\ \text { Fibroadenoma } & 25(25.3) \\ \text { Fibrocystic diseases } & 14(14.1) \\ \text { Phyllodes tumour } & 4(4.0) \\ \text { Lactational Mastitis } & 8(8.1) \\ \text { Intraductal papilloma } & 2(2.0) \\ \text { DCIS } & 1(1.0) \\ \text { Invasive ductal carcinoma } & 43(43.4) \\ \text { Invasive lobular carcinoma } & 2(2.0)\end{array}$

*One patient with benign breast lesion (mastitis) on CNB did not have surgical excision BI-RADS®: Breast Imaging Reporting and Data System; DCIS: Ductal carcinoma in-situ 
Table 2. Profile of indeterminate lesions

\begin{tabular}{|c|c|c|c|c|c|c|}
\hline $\begin{array}{l}\text { CNB } \\
\text { diagnosis }\end{array}$ & $\begin{array}{l}\text { BIRADS } \\
\text { grade }\end{array}$ & $\begin{array}{l}\text { Open surgical } \\
\text { diagnosis }\end{array}$ & $\begin{array}{l}\text { Mean size } \\
\text { (SD) in } \mathrm{cm}^{*}\end{array}$ & $\begin{array}{l}\text { Minimum } \\
\text { (cm)* }\end{array}$ & $\begin{array}{l}\text { Maximum } \\
(\mathrm{cm})^{*}\end{array}$ & $\begin{array}{c}\text { Median } \\
(\mathrm{cm})^{*}\end{array}$ \\
\hline$N$ & 2 & FCD & $5.35(3.19)$ & 2.01 & 11.4 & 3.9 \\
\hline$N$ & 2 & FCD & & & & \\
\hline$N$ & 4 & IDC & & & & \\
\hline$N$ & 5 & IDC & & & & \\
\hline I & 3 & Chronic mastitis & & & & \\
\hline I & 3 & FCD & & & & \\
\hline I & 2 & Fibroadenoma & & & & \\
\hline I & 2 & IDC & & & & \\
\hline I & 4 & IDC & & & & \\
\hline
\end{tabular}

Table 3. Diagnostic validities of clinical examination and US guided CNB for detection of malignant breast lumps

\begin{tabular}{|c|c|c|c|c|c|}
\hline & CE & US & CNB* & CNB** & TA \\
\hline Sensitivity & 93.33 & 95.8 & 84.78 & 92.86 & 100.0 \\
\hline Specificity & 79.63 & 80.39 & 96.23 & 95.83 & 61.11 \\
\hline FPR & 20.4 & 17.85 & 3.77 & 4.17 & \\
\hline FNR & 6.5 & 4.65 & 15.22 & 7.14 & - \\
\hline PPV & 79.25 & 82.14 & 95.12 & 95.12 & 68.18 \\
\hline NPV & 93.48 & 95.34 & 87.93 & 93.88 & 100.0 \\
\hline ODA & 85.86 & 87.88 & 90.90 & 94.44 & \\
\hline
\end{tabular}

CE: Clinical examination; US: Ultrasound; CNB: Core needle biopsy; TA: triple assessment; FPR: False positive rate; FNR: False negative Rate; PPV: Positive predicted value; NPV: Negative predicted value; US: Ultrasound; ODA: overall diagnostic accuracy.

*indeterminate lesions were assumed to be negative for malignancy

**indeterminate lesions excluded

\section{Table 4. Published series of CNB}

\begin{tabular}{|c|c|c|c|c|c|c|c|c|c|}
\hline \multirow[b]{2}{*}{ Author } & \multicolumn{9}{|c|}{ Values in percentage } \\
\hline & Year* & $\mathbf{N}$ & TPR & TNR & FPR & FNR & PPV & NPV & ODA \\
\hline Present study & & 100 & 92.86 & 95.83 & 4.17 & 7.14 & 95.12 & 93.88 & 94.44 \\
\hline Zhou et al. (9) & 2014 & 955 & $92.4 / 92.8+$ & - & - & 1.4 & - & - & - \\
\hline Nagar et al. (10) & 2012 & 162 & 100 & 90 & - & - & 93 & - & - \\
\hline Brancato et al. (11) & 2012 & 1283 & 93.8 & 88.3 & - & 1.7 & - & - & 84.5 \\
\hline Lacambra et al. (12) & 2012 & 464 & 96 & 99 & - & - & 99 & 94 & - \\
\hline Wei et al. (13) & 2011 & 1431 & 88 & 98 & - & - & - & - & 89 \\
\hline Schueller et al. (14) & 2008 & 698 & 95.8 & - & - & 1.6 & - & - & - \\
\hline Luechakiettisak et al. (15). & 2008 & 92 & 92 & 100 & & 7.6 & 100 & 46 & 92 \\
\hline
\end{tabular}




\section{Table 5. Acceptance and complications rates of CNB}

\begin{tabular}{|c|c|c|c|c|c|}
\hline \multicolumn{2}{|l|}{ Degree of acceptance } & $\begin{array}{l}\text { No complications } \\
\qquad N=73\end{array}$ & $\begin{array}{l}\text { Complications } \\
\qquad N=13\end{array}$ & $\begin{array}{l}\text { Total } \\
\mathrm{N}=86\end{array}$ & $\mathbf{p}$ \\
\hline \multicolumn{2}{|l|}{ Fair } & - & $3(23.08)$ & $3(3.49)$ & 0.831 \\
\hline \multicolumn{2}{|l|}{ Good } & $15(20.55)$ & $8(61.54)$ & $23(26.74)$ & \\
\hline \multicolumn{2}{|l|}{ Very good } & $2(2.74)$ & $2(15.38)$ & $4(4.65)$ & \\
\hline \multirow{2}{*}{\multicolumn{2}{|c|}{ Excellent }} & $56(76.71)$ & - & $56(65.11)$ & \\
\hline & & Benign & Malignant & & \\
\hline \multirow[t]{2}{*}{ Recommend to friends and relatives } & Yes & $46(100)$ & $40(100)$ & $86(100)$ & 1.000 \\
\hline & No & - & - & - & \\
\hline \multirow[t]{2}{*}{ Will accept a repeat procedure } & Yes & $46(100)$ & $40(100)$ & $86(100)$ & \\
\hline & No & - & - & - & \\
\hline
\end{tabular}

\section{Discussion}

These findings suggest that our team met its expectations of high overall diagnostic accuracy, specificity, sensitivity and low false-negative rates of ultrasound-guided CNB. These findings are comparable with other previously published series in the literature as shown in Table 4.

In this study, 9 breast lumps were diagnosed as indeterminate lesions, thus giving a non-diagnostic rate of $9.0 \%$ and a false negative rate of $7.14 \%$. Both are within the rate recommended by NHS Breast Cancer Screening Programmes (NHSBSP) (16). Some of the suggested reasons that may account for unsatisfactory sampling include the nature of the lesion such as a radial scar or complex sclerosing adenosis and error in sampling technique (17). In the present study, we noticed that all the indeterminate cases were recorded at the initial part of the study, suggesting that the accuracy improves as the volume of procedures increase.

The recorded false-positive rates in this study (FPR) of $4.17 \%$ was higher than the recommended rate by the NHSBSP16. NHSBSP recommends that the minimum value for FPR is $0.5 \%$. The high value of FPR recorded in this study was undesirable. This may have been accounted for by initial interpretation errors by the pathologist suggesting the need for independent diagnosis by at least two breast pathologists. This will help to maximise the detection of malignancy and achieve a high level of accuracy and consistency in reporting breast lesions.

With the exclusion of the indeterminate lesions, ultrasound-guided CNB showed sensitivity and specificity of $92.8 \%$ and $95.83 \%$ respectively with an overall diagnostic accuracy of $94.44 \%$. The sensitivity, specificity and diagnostic accuracy recorded in this study were comparable with findings in similar studies (9-15). The overall success rate recorded in this study further underscores the importance of a team approach in the evaluation of breast lesions.

The agreement between the histopathological subclassification of breast masses into definite histological entities using ultrasound-guided CNB specimens and open surgical specimens was analysed using unweighted kappa- coefficient and at a 95\% confidence interval. The k-coefficient values were 0.798 ( $95 \%$ confidence interval of 0.69 to 0.90 ) and 0.801 ( $95 \%$ confidence interval of 0.71 to 0.92 ) with $\mathrm{p}<0.05$ for benign and malignant breast lumps respectively. This $\mathrm{k}$ value of $>0.7$ shows substantial agreement (18) between the two pathological results and this was significantly greater than zero in this study $(0=$ no agreement). Zhou et al. (9) also recorded similar high kappa value. Nevertheless, this kappa value is still less than 1(perfect agreement). This finding is within the acceptable range recommended by NHSBSP (16). Achieving a perfect agreement has been elusive despite advances in biopsy devices and techniques. Factors that may limit the accuracy of CNB in identifying the specific histology include certain conditions like Fibroepithelial lesions with cellular stroma and phyllodes tumours, papillary lesions, mucinous lesions, radial scars and atypical proliferative lesions. In this study, two cases of ductal carcinoma insitu recorded on ultrasound-guided CNB sample histology were later found to be invasive on histopathological examination of the surgical specimen. This agrees with other reports in literature (19). Knowledge of this is important particularly for women contemplating whether or not to undergo surgery for DCIS. Though, in our study, we observed that the majority of the women opted for surgical excision of breast lumps even when they are benign. Only one patient with chronic mastitis diagnosed after CNB accepted to be followed up.

Ultrasound-guided CNB is generally regarded as a safe procedure and associated with few insignificant complications. The recorded few complications in this study included pain at the site of procedure which was relieved by the intake of oral analgesics (paracetamol) and breast hematoma. This concurs with findings in the literature (20). All the patients that had breast hematoma had malignant breast lumps. This is most likely due to increased vascularity associated with malignant conditions. In our standard practice, patients do not routinely undergo ultrasound post-biopsy so the results in this study may likely underestimate the true incidence of hematoma formation. It is reasonable, however, to conclude that no clinically significant complication occurred. For patients who are on anticoagulants or antiplatelet medications, FNAC (fine needle aspiration cytology) has been found to have reduced risk of bleeding than $\mathrm{CNB}$ though, Melotti et al. (21) did not record any significant bleeding when they carried out CNB in patients on anticoagulants and antiplatelets.

Finally, ultrasound-guided CNB was found to be acceptable among the patients that underwent the procedure even among patients with complication. All the patients in the current study said they would recommend the procedure to relatives and friends that have a similar condition. They were all willing for a repeat procedure in case of recurrence or 
new case of a breast lump. This suggests that $\mathrm{CNB}$ is well accepted by the patients as reported in the literature (22). This high level of acceptance among the patients in this study may be due to absence of major complications during the procedure, or because the procedure was done at no extra cost to the patients care. Probably the response may be different if there were additional costs due to the ultrasound investigations.

There are certain limitations in the present study. First, this study was the preliminary experience in a breast clinic run by the multidisciplinary team. Technique errors and wrong interpretations experienced in the early phase of the work affected the various diagnostic parameters assessed particularly the high false-negative rates and false-positive rates compared with previous studies shown in Table 3. Further evaluation is necessary to report the actual sensitivity and specificity after the initial learning curve. Secondly, our team relied on a surgeon with prior experience on freehand guided breast biopsy which may have lowered the effectiveness of the initial procedures. In spite of these limitations, our study showed high concordance between CNB and open surgical specimens and reduced our waiting time for diagnosis

In conclusion, we have demonstrated that $16 \mathrm{G}$ ultrasound-guided $\mathrm{CNB}$ can be used as a reliable diagnostic alternative to surgical biopsy even in the absence of formal training to facilitate diagnostic evaluations of palpable breast lesions. The multidisciplinary breast care team that has successfully met its objectives of prompt accurate diagnosis also showed the need for further training of the members. We believed that competence in this procedure requires at minimum 40 samplings and there is a need for two pathologists to view the CNB specimens in the initial part of this procedure until competence is acquired. Team approach to breast diseases diagnosis is possible even in a resource-limited country like $\mathrm{Ni}$ geria. CNB is a relatively safe procedure, and well tolerated by patients with minimal complications with a high acceptance rate.

Ethics Committee Approval: Ethics committee approval was received for this study from the ethics committee of Nnamdi Azikiwe University Teaching Hospital Nnewi (24.06.2014/ NAUTH/CS/66/VOL.6/4)

Informed Consent: Written informed consent was obtained from the patients who participated in this study.

Peer-review: Externally peer-reviewed.

Author Contributions: Concept - C.O., S.A.; Design - U.E., C.U., M.O.; Supervision - G.C., S.A., C.U.; Resources - C.E., E.U.; Materials - S.A., E.U.; Data Collection and/or Processing - C.O., O.E., E.I.; Analysis and/or Interpretation - C.O., E.I.; Literature Search - C.O., C.E.; Writing Manuscript C.O., O.E.; Critical Review - S.A., G.C., U.E.; Other - M.O., E.U.

Acknowledgements: We would like to thank the staff of Department of Radiology, Pathology, Surgery and Nnamdi Azikiwe University Tumour Board.

Conflict of Interest: The authors have no conflicts of interest to declare.

Financial Disclosure: The authors declared that this study has received no financial support.

\section{References}

1. Rajan S, Foreman J, Wallis MG, Caldas C, Britton P. Multidisciplinary Decisions in Breast Cancer: Does the Patient Receive What the Team Has Recommended? Br J Cancer 2013; 108: 2442. (PMID:23736032) [CrossRef]

2. Association of Breast Surgery at Baso 2009. Surgical Guidelines for the Management of Breast Cancer. Eur J Surg Oncol. 2009; 35: 1-22. (PMID:19299100) [CrossRef]
3. Selby P, Gillis C, Haward R. Benefits from Specialised Cancer Care. Lancet 1996; 348: 313-318. (PMID: 8709693) [CrossRef]

4. Nzeako HC. Evaluation of Core Needle Biopsy in the Diagnosis of Palpable Breast Masses in Nnamdi Azikiwe University Teaching Hospital, Nnewi Dissertation for Fellowship of the Medical College in Surgery. Lagos: National Postgraduate Medical College of Nigeria. 2014.

5. Shulman LN, Willett W, Sievers A, Knaul FM. Breast Cancer in Developing Countries: Opportunities for İmproved Survival. J Oncol 2010 Dec 29. doi: 10.1155/2010/595167. [Epub ahead of print] (PMID: 21253541) [CrossRef]

6. Okoli C, Anyanwu SN, Ochomma AO, Emegoakor CD, Chianakwana GU, Nzeako H, et al. Assessing the Quality of Life of Patients with Breast Cancer Treated in a Tertiary Hospital in a Resource-Poor Country. World J Surg 2018; 27: 1-8. (PMID:30151677) [CrossRef]

7. Apesteguía L, Pina LJ. Ultrasound-guided Core-needle Biopsy of Breast Lesions. Insights Imaging 2011; 2: 493-500 (PMID: 22347970) [CrossRef]

8. Van Breest Smallenburg V, Nederend J, Voogd AC, Coebergh JW, Van Beek $\mathrm{M}$, Jansen FH, et al. Trends in Breast Biopsies for Abnormalities Detected at Screening Mammography: A Population-based Study in the Netherlands. Br J Cancer 2013; 109: 242. (PMID: 23695018) [CrossRef]

9. Zhou JY, Tang J, Wang ZL, Lv FQ, Luo YK, Qin HZ, et al. Accuracy of 16/18G Core Needle Biopsy for Ultrasound-visible Breast Lesions. World J Surg Oncol 2014; 12: 1-7. (PMID: 24400744) [CrossRef]

10. Nagar S, Iacco A, Riggs T, Kestenberg W, Keidan R. An Analysis of Fineneedle Aspiration Versus Core Needle Biopsy in Clinically Palpable Breast Lesions: A Report on the Predictive Values and a Cost Comparison. Am J Surg 2012; 204: 193-198. (PMID: 22464444) [CrossRef]

11. Brancato B, Crocetti E, Bianchi S, Catarzi S, Risso GG, Bulgaresi P, et al. Accuracy of Needle Biopsy of Breast Lesions Visible on Ultrasound: Audit of Fine Needle Versus Core Needle Biopsy in 3233 Consecutive Samplings with Ascertained Outcomes. Breast 2012; 21: 449-454 (PMID: 22088803) [CrossRef]

12. Lacambra MD, Lam CC, Mendoza P, Chan SK, Alex MY, Tsang JY, et al. Biopsy Sampling of Breast Lesions: Comparison of Core needle-and Vacuum-assisted Breast Biopsies. Breast Cancer Res Treat 2012; 132:917923. (PMID: 21698409) [CrossRef]

13. Wei X, Li Y, Zhang S, Zhu Y, Fan Y. Experience in Large-core Needle Biopsy in the Diagnosis of 1431 Breast Lesions. Med Oncol 2011; 28: 429-433. (PMID:20339957) [CrossRef]

14. Schueller G, Schueller-Weidekamm C, Helbich TH. Accuracy of Ultrasound-guided, Large-core Needle Breast Biopsy. Eur Radiol 2008; 18: 1761 (PMID: 18414872) [CrossRef]

15 Luechakiettisak P, Rungkaew P. Breast Biopsy: Accuracy of Core Needle Biopsy Compared with Excisional or Incisional Biopsy: A Prospective Study. Thai Journal of Surgery 2008; 29: 6-10.

16. Lee AH, Carder P, Deb R, Howe M, Knox F, Shrimankar J, et al. Guidelines for Non-operative Diagnostic Procedures and Reporting in Breast Cancer Screening. London, UK: The Royal College of Pathologists. 2016.

17. Mitra S, Dey P. Fine-needle Aspiration and Core Biopsy in the Diagnosis of Breast Lesions: A Comparison and Review of the Literature. Cytojournal 2016; 13:18. (PMID: 27651820) [CrossRef]

18. Landis JR, Koch GG. The Measurement of Observer Agreement for Categorical Data. Biometrics. 1977; 1:159-174 (PMID: 843571) [CrossRef]

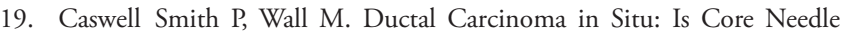
Biopsy Ever Enough? J Med Imaging Radiat Oncol 2017; 61: 29-33 (PMID: 27554420) [CrossRef]

20. Parker SH, Burbank F, Jackman RJ, Aucreman CJ, Cardenosa G, Cink TM, et al. Percutaneous Large-core Breast Biopsy: A Multi-institutional Study Radiology. 1994; 193: 359-364. (PMID: 7972743) [CrossRef]

21. Melotti MK, Berg WA. Core Needle Breast Biopsy in Patients Undergoing Anticoagulation Therapy: Preliminary Results. AJR Am J Roentgenol. 2000; 174: 245-249 (PMID: 10628487) [CrossRef]

22. Park HL, Kim LS. The Current Role of the Vacuum-assisted Breast Biopsy System in Breast Disease. J Breast Cancer. 2011; 14: 1-7 (PMID: 21847387) [CrossRef] 